ARTICLE

\title{
CFTR trafficking mutations disrupt cotranslational protein folding by targeting biosynthetic intermediates
}

Hideki Shishido (1) ', Jae Seok Yoon ${ }^{1}$, Zhongying Yang ${ }^{2} \&$ William R. Skach (10) ${ }^{3 凶}$

Protein misfolding causes a wide spectrum of human disease, and therapies that target misfolding are transforming the clinical care of cystic fibrosis. Despite this success, however, very little is known about how disease-causing mutations affect the de novo folding landscape. Here we show that inherited, disease-causing mutations located within the first nucleotide-binding domain (NBD1) of the cystic fibrosis transmembrane conductance regulator (CFTR) have distinct effects on nascent polypeptides. Two of these mutations (A455E and L558S) delay compaction of the nascent NBD1 during a critical window of synthesis. The observed folding defect is highly dependent on nascent chain length as well as its attachment to the ribosome. Moreover, restoration of the NBD1 cotranslational folding defect by second site suppressor mutations also partially restores folding of full-length CFTR. These findings demonstrate that nascent folding intermediates can play an important role in disease pathogenesis and thus provide potential targets for pharmacological correction.

${ }^{1}$ CFFT Lab, Cystic Fibrosis Foundation, 44 Hartwell Ave, Lexington, MA 02421, USA. ${ }^{2}$ Department of Chemical Physiology and Biochemistry, Oregon Health \& Science University, 3181 S.W. Sam Jackson Park Rd, Portland, OR 97239, USA. ${ }^{3}$ Cystic Fibrosis Foundation, 4550 Montgomery Ave., Suite 1100 , Bethesda, MD 20814, USA. ${ }^{凶}$ email: wskach@cff.org 
C ystic fibrosis (CF) is a lethal genetic disease that is primarily caused by misfolding of the cystic fibrosis transmembrane conductance regulator (CFTR), a polytopic membrane protein with two six-spanning transmembrane domains (TMD1, TMD2), two nucleotide-binding domains (NBD1, NBD2), and a relatively unstructured regulatory (R) domain $^{1}$. Of the $>1700$ genetic variants reported in the CF gene (www.genet.sickkids.on.ca/cftr), several hundred lead to loss of chloride transport by disrupting CFTR folding and intracellular trafficking ${ }^{1-3}$. The most common disease-associated allele, a three base pair deletion in NBD1 (F508del), is found in $~ 87 \%$ of people with CF where it disrupts CFTR folding by at least two mechanisms: (i) directly inhibiting NBD1 folding efficiency and/ or stability ${ }^{4-6}$; and (ii) destabilizing an intramolecular interface between NBD1 and the 4th intracellular loop (ICL4) in TMD2 ${ }^{6-8}$. Over the past two decades, high-throughput screening efforts have identified multiple small molecules, often referred to as corrector molecules, with the ability to partially restore normal F508del CFTR folding in cells ${ }^{9}$, and three such molecules have recently received FDA approval as components of combination drugs to treat $\mathrm{CF}^{10-13}$. At present, however, the molecular mechanism(s) by which other inherited mutations disrupt CFTR folding and trafficking remain largely unknown.

For CF and other human diseases caused by aberrant protein folding, it has been difficult to experimentally determine precisely when a mutant protein deviates from its native folding pathway. For example, although mutations have been shown to influence folding intermediates immediately after synthesis ${ }^{14}$, little is known about whether misfolding occurs during synthesis itself. This is particularly relevant because the CFTR corrector molecules lumacaftor and presumably tezacaftor, must be present during CFTR synthesis to optimally stimulate CFTR folding in cells, suggesting that they might act on a transient folding intermediate ${ }^{15}$. In addition, protein folding in cells is coupled to translation as the nascent polypeptide emerges from the ribosome exit tunnel ${ }^{16-26}$. De novo folding is therefore influenced by numerous factors ${ }^{25,26}$, including the presence of the adjacent ribosome $\mathrm{e}^{18,20,22,25-30}$, translation elongation rate $\mathrm{r}^{18,19,25,26,31-34}$, molecular crowding 35,36 , and interaction with cellular chaperones $25,26,37-42$. Thus, an important question in understanding basic mechanisms of folding disorders is whether disease-causing mutations prevent the initial acquisition of native structure by disrupting the cotranslational folding landscape.

CFTR folding is intrinsically complex and involves insertion of 12 transmembrane helices into the lipid bilayer, individual folding of soluble domains, and assembly of these domains into the mature protein structure. Some of these steps occur cotranslationally during synthesis, whereas others occur posttranslationally. NBD1 folding is a critical step in this process, and the efficiency of NBD1 folding is a limiting step in CFTR biosynthesis $8,43,44$. In addition, NBD1 does not spontaneously refold in vitro ${ }^{4,5}$, suggesting that the de novo protein folding pathway has a critical role in achieving a native structure. Genetic suppressor mutations that improve NBD1 folding efficiency or ICL4-NBD1 interactions can partially restore intracellular trafficking of F508del and certain other CFTR mutants ${ }^{6-8}$, whereas correction of both defects restores folding to near wild-type levels. These results suggest that therapeutic agents, which improve cotranslational folding efficiency, could provide a pharmacological strategy for treating protein folding disorders.

To better understand how CFTR, and proteins in general, fold in cells, we devised a strategy to interrogate nascent polypeptides as they are synthesized on the ribosome ${ }^{17,18}$. Using this system, we previously showed that NBD1 acquires its structure cotranslationally through a distinct series of carefully choreographed folding events. Folding is initiated by rapid compaction of an
N-terminal subdomain (CFTR residues \#389-491), and then followed by folding of the $\alpha$-helical subdomain (residues $500-564$ ), and a parallel four-stranded $\beta$-sheet that is buried in the core of the domain (residues 568-603). Although synthesis of these subdomains proceeds sequentially, $\mathrm{N}$-terminus to $\mathrm{C}$-terminus, the timing of these folding events is tightly coordinated to ensure that $\alpha$-helical subdomain collapse is delayed until the $\beta$ sheet core is synthesized.

In the current study, we examine how CF disease-causing mutations impact these cotranslational folding events at defined stages of NBD1 synthesis. We show that: (1) numerous NBD1folding mutations impact nascent polypeptide folding; (2) two mutations (A455E and L558S) in different regions of NBD1 delay cotranslational compaction of folding intermediates during a similar window of synthesis of the $\beta$-sheet core; (3) destabilizing effects of the ribosome augment the mutation-induced folding defect; and (4) correction of this transient defect via second site suppressor mutations partially restores trafficking of full-length CFTR in cells. These studies indicate that de novo folding events can impact final folding outcome and provide insight into how disease-causing mutations contribute to protein folding disorders by perturbing intermediate folding states that transiently populate the folding pathway.

\section{Results}

CF mutations perturb cotranslational folding intermediates. Numerous missense mutations that disrupt CFTR folding have been identified within CFTR NBD1 subdomains, including the Nterminal subdomain (L441P and A455E), the N-terminal/a-helical subdomain interface (S492F), and the $\alpha$-helical subdomain (I507del, F508del, V520F, L558S, A559T, R560K, and R560T) 2,45 (Fig. 1a). To determine whether these mutations influence the cotranslational folding pathway, we used fluorescence resonance energy transfer (FRET) to monitor the overall state of protein compaction as the nascent polypeptide is synthesized on the ribosome (Fig. 1b). This approach involves inserting two fluorescent molecules consisting of an $\mathrm{N}$-terminal donor fluorophore, cyan fluorescent protein (CFP), and an internal small acceptor dye (7-nitrobenz-2-oxa-1,3-diazole) into the nascent polypeptide in 1:1 stoichiometry during synthesis ${ }^{46-48}$ (Fig. 1b, c \& Supplementary Fig. 1a-c). Because protein folding principally involves compaction of an extended polypeptide, many residues that are distant in primary structure are brought into close proximity as native structure is acquired ${ }^{48}$. For appropriate donor and acceptor probe sites, an increase in FRET efficiency therefore provides a measure of compaction as the nascent polypeptide grows in length and transitions from an elongated random (unfolded) conformation to a compacted (folded) conformation ${ }^{17,18,49}$.

To examine NBD1 nascent folding intermediates, in vitrotranscribed RNA was truncated in the NBD1-coding sequence and expressed in a rabbit reticulocyte lysate (RRL) cell-free translation system. The terminal stop codon was removed by RNA truncation, which stably binds the nascent polypeptide via a peptidyl-tRNA bond to tRNA at the ribosome P-site, thus generating a uniform cohort of nascent ribosome-attached polypeptides that can be isolated and analyzed ${ }^{17,18,46,47}$ (Supplementary Fig. 1a-c). We previously showed that when nascent NBD1 is synthesized in this system, it folds into a native-like structure that is capable of binding $\mathrm{ATP}^{17}$. We also identified several permissive sites for acceptor dye incorporation that do not grossly perturb NBD1 folding ${ }^{17,18}$. The ability to monitor NBD1 folding while attached to the ribosome thus allows access to native folding intermediates that exist only transiently during CFTR synthesis. By translating a series of RNA transcripts 
a

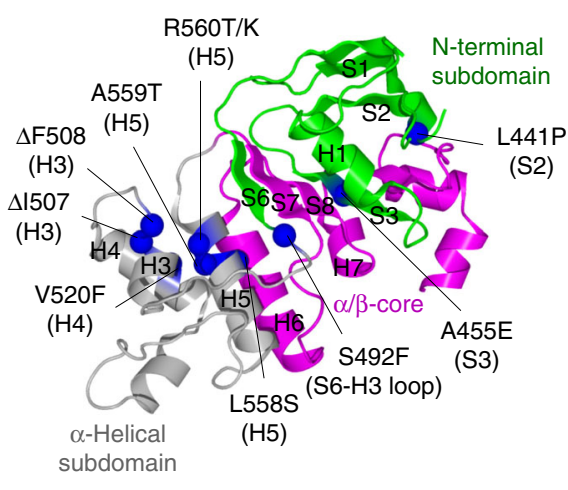

b
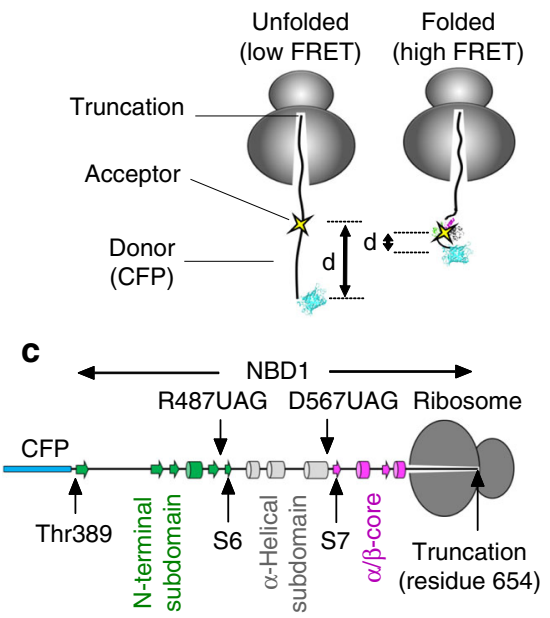

e

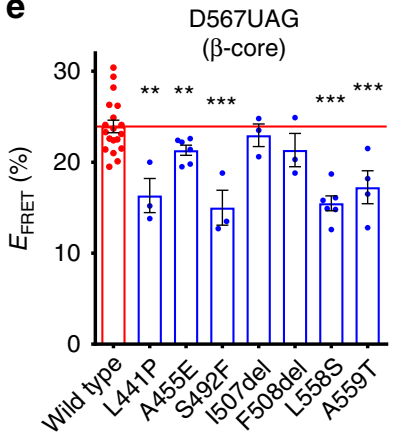

Fig. 1 CF-causing mutations perturb NBD1 biosynthetic intermediates. a Location of CFTR processing mutations shown in the NBD1 structure taken from the human CFTR Cryo-EM structure (PDB: 5 UAK). b Cartoon conceptually depicting FRET assay using translationally incorporated fluorophores to detect structural transitions of ribosome-attached nascent polypeptides. c Schematic of ribosome-attached CFP-NBD1 fusion protein truncated at CFTR residue 654 showing locations of FRET acceptor dye at CFTR residues Arg487, or Asp567, which report folding of the $\alpha$-helical subdomain, or $\beta$-sheet core, respectively ${ }^{17,18}$. Helices and $\beta$-strands are drawn as cylinders and filled arrows, respectively. d, e FRET efficiencies obtained for wild-type and mutant nascent polypeptides in (ribosome-attached) CFP-NBD1 constructs truncated at residue 654 and containing acceptor dye at residue Arg487 d or Asp567 e. Each dot represents data from an independent experiment. Each bar shows mean $\pm \mathrm{SEM}, n \geq 3$ independent experiments. Two-tailed student's $t$ test comparing wild-type and indicated mutant, asterisk $p<0.05$, double asterisk $p<0.01$, triple asterisk $p<0.001$, n.s. $>0.05$ otherwise indicated. Red line shows mean of FRET efficiency for wild-type. Source data of $\mathbf{d}$ and $\mathbf{e}$ are provided as a Source Data file.

truncated at defined lengths, it is possible to capture kinetically stalled translation intermediates that reflect snapshots of the folding process and thus to reconstruct the relative compaction of different subdomains as a function of nascent polypeptide length ${ }^{17,18,47,48,50,51}$. Moreover, RRL mimics the folding environment of the cell in that it includes a broad range of cellular chaperones $^{52}$ (e.g., HSP40, HSP70, HSP90, etc.), degradation machinery (ubiquitin proteasome pathway) ${ }^{53}$, and molecular crowding ( $20 \%$ cytosol $\sim 80 \mathrm{mg} / \mathrm{ml}$ protein). An important caveat is that truncated polypeptides may lack critical native stabilizing contacts and therefore represent a structural ensemble rather than a predominant stable structural intermediate. Truncated cohorts are therefore referred to simply as nascent polypeptides or folding intermediates. Structural perturbations detected by changes in FRET may thus reflect changes in the ensemble that ultimately gives rise to the final folded structure.

Initial studies were carried out using nascent polypeptides truncated at CFTR residue 654 with the acceptor dye inserted either at residue Arg487 (near the S6 $\beta$-strand) or Asp567 (near the S7 $\beta$-strand), which report on folding of the $\alpha$-helical subdomain, and the $\beta$-sheet core, respectively ${ }^{18}$ (Fig. 1c, Supplementary Fig. 1a). This truncation represents a critical intermediate in which the $\beta$-sheet core is in the process of acquiring its native structure ${ }^{18}$, which in turn is temporally coupled to $\alpha$-helical subdomain compaction ${ }^{18}$. Mutations that alter the timing of $\alpha$-helical subdomain compaction for this intermediate state would therefore most likely negatively impact CFTR folding. As shown in Fig. 1d, several, but not all trafficking mutations decreased $\alpha$-helical subdomain compaction as measured by small but significant decreases in FRET efficiency for nascent chains containing the acceptor dye at Arg487. An overlapping set of mutations, L441P, A455E, S492F, L558S, and A559T, but not I507del or F508del, decreased compaction of the $\beta$-sheet core (Fig. 1e). Thus, multiple trafficking mutations can cotranslationally perturb NBD1-folding intermediates. As NBD1 folding involves a complex series of steps, it is perhaps not surprising that some mutations do not affect the cotranslational pathway, but exert their effect elsewhere. As we showed previously, F508del had little effect on FRET using this assay ${ }^{18}$, which could indicate either that, (i) it does not affect NBD1 translation intermediates per se, (ii) changes were not detected by these specific probe sites, or (iii) changes were masked by the reduced translation temperature $\left(24^{\circ} \mathrm{C}\right)$, which is known to suppress the folding defect. 
a

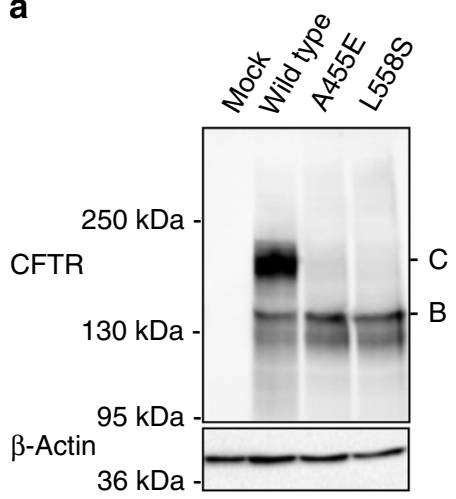

C
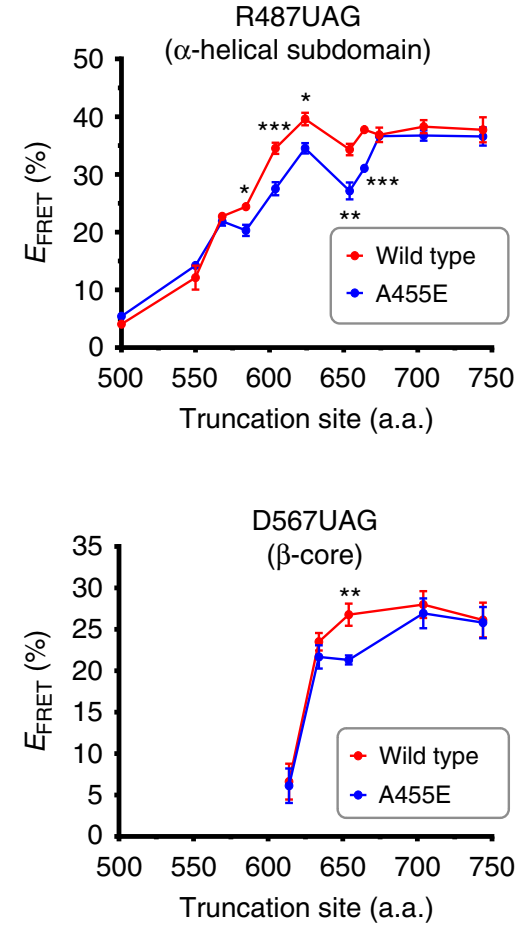

b

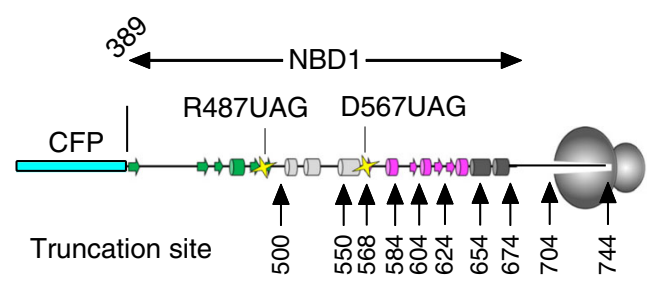

d

R487UAG

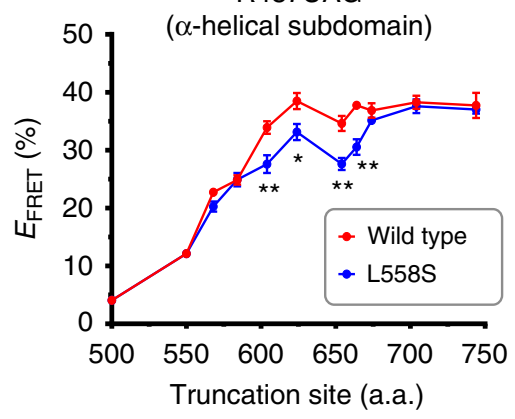

f

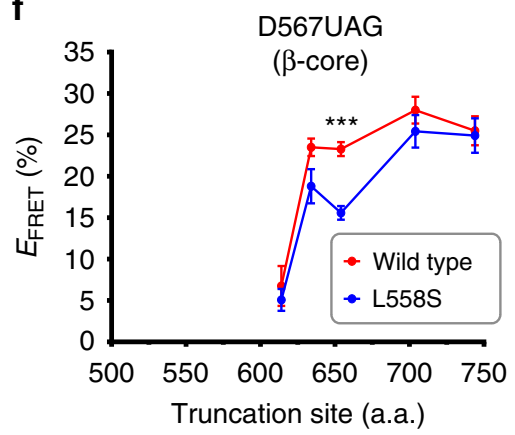

Fig. 2 A455E, L558S transiently disrupt NBD1 cotranslational folding. a Immunoblot of full-length CFTR (wild-type, A455E, or L558S) expressed in human embryonic kidney (HEK) 293 cells showing core glycosylated (band B) and mature CFTR (band C). Uncropped blots in Source Data. b Schematic of ribosome-attached CFP-NBD1 showing approximate location of acceptor dye incorporation sites (residue Arg487 or Asp567) and truncation sites used in c-f. c-f Length-dependent FRET efficiencies for wild-type (red line) and A455E or L558S (blue line) obtained from ribosome-attached CFP-NBD1 constructs with acceptor dye located at R487UAG c, d or D567UAG e, f for each truncation site indicated. Data are mean \pm SEM, $n \geq 3$ independent experiments. Two-tailed student's $t$ test comparing wild-type and A455E or L558S, asterisk $p<0.05$, double asterisk $p<0.01$, triple asterisk $p<0.001$, n.s. $>0.05$ otherwise indicated. Note that wild-type data for truncation sites 500,568, 584, and 664, and A455E data for truncation sites 500, 550, 568, 664 shown in c, and wild-type data for truncation sites 500,550,568,584, and 664, and L558S data for truncation site 664 shown in $\mathbf{d}$ have error bars smaller than the dots. Some data for truncation sites $568,604,654,666,704$, and 744 shown in c, d are also taken from Figs. 1d, 3a, b, 4a, b, \& 5b. Source data of a, c-f are provided as a Source Data file.

A455E and L558S transiently alter NBD1 folding pathway. Three mutations, A455E, L558S, and A559T, demonstrated statistically significant folding disruptions of both a-helical subdomain and $\beta$-sheet core (Fig. 1d, e). As L558S showed a larger change in FRET than A559T, we focused subsequent studies on A455E and L558S, located within the N-terminal and $\alpha$-helical subdomains, respectively. Both of these mutations induce a severe block in CFTR trafficking to the cell surface (Fig. 2a), consistent with previous studies ${ }^{2}$. We next tested the effects of A455E and L558S on nascent chains of increasing length generated from sequentially truncated RNA transcripts (Fig. 2b). Wild-type nascent polypeptides with an acceptor dye located at Arg487 underwent a gradual compaction of the a-helical subdomain during synthesis of residue 500 to residue 624 (FRET efficiency increase from $4.1 \pm 0.8 \%$ to $39.6 \pm 1.1 \%$ (mean \pm SEM, $n \geq 3$ independent experiments), respectively (Fig. 2c, d), as previously reported ${ }^{18}$. For the A455E mutant, a decrease in FRET $(4.1 \pm 1 \%$ decrease) from that of wild-type was first observed at truncation 584, suggesting that compaction of these nascent polypeptides deviates from wild-type $\sim 100$ residues after the mutant residue is synthesized (Fig. 2c). In contrast, the effect of L558S (located in H5) on the $\alpha$-helical subdomain was first observed at truncation 604, almost immediately after the mutant residue entered the cytosol (6.3 $\pm 2.0 \%$ decrease) (Fig. 2d). The most profound effect of both mutations on the a-helical subdomain compaction was observed at truncation $654(7.2 \pm 0.9 \%$ decrease for A455E and 
$7.0 \pm 1.0 \%$ decrease for L558S). In addition, FRET efficiency approached wild-type levels at truncation residue 674 when NBD1 synthesis was completed ${ }^{18}$. Note that $30-40$ residues of the nascent polypeptide are sequestered within the ribosome exit tunnel. Tertiary folding is therefore limited to cytosolically exposed residues that are similarly offset from the truncation site, and $\mathrm{H} 5$ emerges from the ribosome during synthesis of residues 584-604. Both A455E and L558S therefore disrupt NBD1-folding intermediates during a very specific window of synthesis as $\alpha$ helix $\mathrm{H} 5$ to $\beta$-strand S7 emerge from the ribosome. Because $\mathrm{H} 5$ is the location of numerous CFTR-trafficking mutations as well as second site suppressor mutations that partially correct F508del CFTR folding ${ }^{8}$, this region of NBD1 likely makes critical contacts during initial collapse and compaction of the NBD1 core.

In contrast to the $\alpha$-helical subdomain, compaction of the wildtype $\beta$-sheet core occurred more abruptly and at a slightly later stage of synthesis (between truncations 614-654) (Fig. 2e, f). Both A455E and L558S also disrupted $\beta$-sheet core folding, again, during a similar and relatively narrow window of synthesis that overlapped with effects on the $a$-helical subdomain (Fig. 2e, f). L558S showed a more prolonged effect and greater change in FRET, suggesting that the mutations alter $\beta$-sheet core folding by somewhat different mechanisms.

Results from Fig. 2 indicate that despite being separated by $\sim 100$ residues in primary sequence, A455E and L558S alter the NBD1 folding landscape during the same period of translation and thus likely act on similar folding intermediate(s). The effect on FRET also diminished as NBD1 synthesis was extended to residue 674 . These findings suggest that both mutations disrupt the coordinated compaction of $\alpha$-helical, and $\beta$-sheet core subdomains. Moreover, the NBD1-folding intermediates appear particularly susceptible to perturbation early during synthesis, but less so as the structure of the domain is stabilized by addition of C-terminal residues. We also analyzed two CFTR variants as negative controls to confirm that results in Fig. 2 are not simply a result of fluctuation of the assay system. M470V is a polymorphism in the N-terminal subdomain that is not associated with disease, whereas G551D, also located in H5, causes CF by disrupting CFTR channel gating but has no effect on folding or trafficking. Neither of these variants produced detectable changes in FRET for any of the critical folding intermediates (truncations 604, 614, and 654, Supplementary Fig. 2a, b), supporting our conclusions that the observed compaction delay is specific for misfolding mutations.

The ribosome accentuates transient folding defects. We previously demonstrated that the ribosome exerts a destabilizing effect on the nascent polypeptide that transiently delays folding of the $a$-helical subdomain, thereby keeping it in a more open conformation during synthesis of the $\beta$-sheet core ${ }^{18}$. Moreover, this delay was required for proper folding and packing of the $\beta$ sheet core as the polypeptide emerges from the ribosome ${ }^{18}$. The current study demonstrates that A455E and L558S further delay folding of both the $\alpha$-helical subdomain and the $\beta$-sheet core and thus alter the normal temporal coupling of these folding events (Fig. 2c-f). We therefore wondered how the tethered ribosome influenced the destabilizing effect of these mutations on nascent polypeptide conformation.

Figure 3 a shows that wild-type and both mutant polypeptides truncated at residue 568 exist in a relatively unfolded state while attached to the ribosome, and all three polypeptides exhibited a substantial increase in FRET following ribosome release. When nascent polypeptides were elongated to truncation 604 , the wildtype polypeptide folded on the ribosome and remained folded following release. In contrast, A455E and L558S mutants remained in a more open conformation under both conditions. This finding is consistent with data from Fig. 2 that both mutations exert a disruptive effect on the native conformation at this chain length.

To specifically determine the effect of the ribosome on A455Eand L558S-induced misfolding, we compared FRET obtained from ribosome-released polypeptides truncated at residue 568 to ribosome-attached polypeptides truncated at residue 604 because at these truncations, equivalent residues are cytosolically exposed and available for tertiary folding. As shown in Fig. 3a, mutant polypeptides were less compact than wild-type polypeptides in the ribosome-attached state. Yet, all three peptides (at truncation 568) yielded the equivalent high FRET values after ribosome release, indicating that ribosome attachment appears to augment this particular mutation-induced folding defect (Fig. 3a, b). In other words, the more open conformation observed for mutants at truncation 604 is accentuated by the destabilizing effect that the ribosome exerts on the nascent polypeptide. Moreover, as polypeptides were elongated to residue 604 , the mutant polypeptides failed to fold normally even after they were released from ribosome (Fig. 3a, b). This suggests that the nascent (attached) polypeptide has been driven into an off-pathway intermediate by the combined disruptive effects of the mutations and the ribosome, resulting in the inability to fold properly if it is released free into solution at this stage of synthesis.

The second key finding in Fig. 2c, d is that as NBD1 synthesis was completed, mutant nascent polypeptides were eventually able to acquire a compact state, but at a longer truncation length than the wild-type protein. Thus, the net effect of the mutations is to further delay $a$-helical compaction beyond the normal folding window. We therefore examined the role of the ribosome in mediating this late-stage folding event.

At truncation 664, ribosome-bound A455E and L558S polypeptides still exhibited a less compacted state than wildtype polypeptides (FRET efficiency $=31.8 \pm 0.4 \%$ for A455E and $32.2 \pm 1.0 \%$ for L558S), and both mutants failed to fold after ribosome release (Fig. 3c). At truncation 704, however, ribosomeattached wild-type and mutant NBD1 nascent polypeptides yielded equivalent high FRET values (FRET efficiency $=38.3 \pm$ $1.1 \%$ for wild-type, $36.8 \pm 0.9 \%$ for $\mathrm{A} 455 \mathrm{E}$, and $37.6 \pm 1.2 \%$ for L558S) (Fig. 3c). Because polypeptides released at truncation 664 have equivalent aqueous exposed residues as the attached polypeptides at truncation 704 (Fig. 3d), ribosome attachment must somehow facilitate eventual compaction of the full-length domain. This result is analogous to the previously reported ribosome effect in wild-type polypeptides ${ }^{18}$, but occurs at a longer chain length for the mutant polypeptides. Taken together, our results indicate that ribosome exerts at least two unexpected effects on the mutant nascent polypeptides. It participates in destabilizing the misfolded state at an early stage of synthesis by delaying compaction of the $\alpha$-helical subdomain and $\beta$-sheet core, and subsequently maintains the growing nascent polypeptide in a non-native, but permissive conformation that allows delayed compaction as additional folding information is provided during completion of synthesis (Fig. 3).

Thermal stability of mutant NBD1-folding intermediates. We next examined the effect of the A455E and L558S on thermal stability of NBD1-folding intermediates by subjecting ribosomebound nascent polypeptides to progressive thermal denaturation. In control experiments we showed that with the acceptor dye located at Thr389 (CFP fusion site) (Supplementary Fig. 3a), CFP-NBD1 (full-length NBD1) exhibited a reversible temperature-dependent decrease in CFP fluorescence but no change in FRET (Supplementary Fig. 3b, c). This was expected as 
a

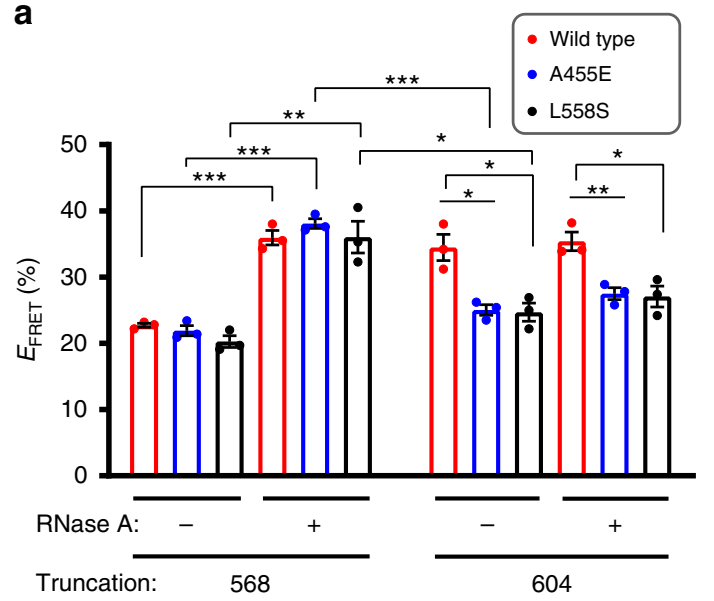

b

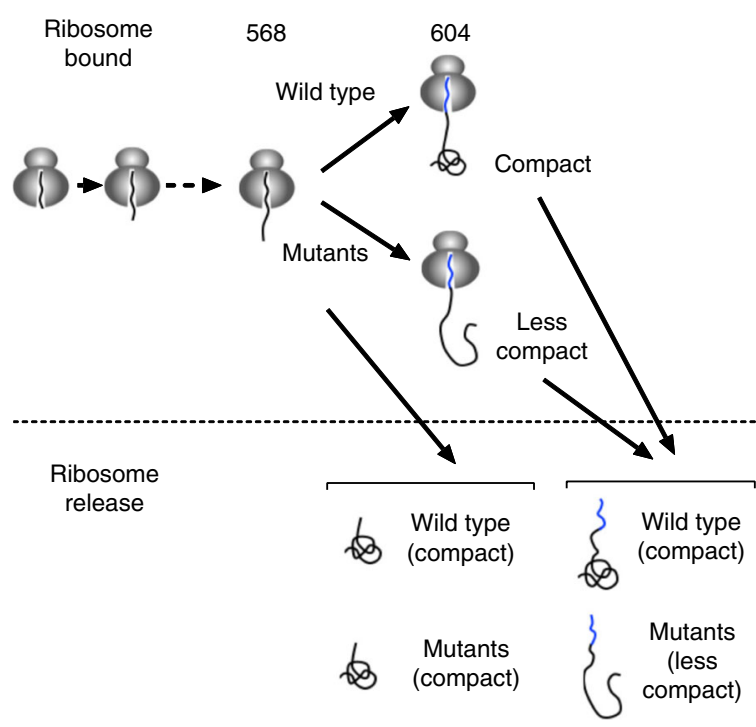

C

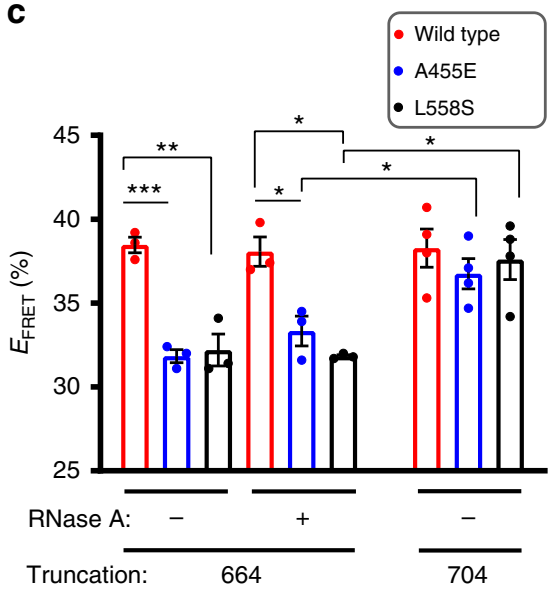

d

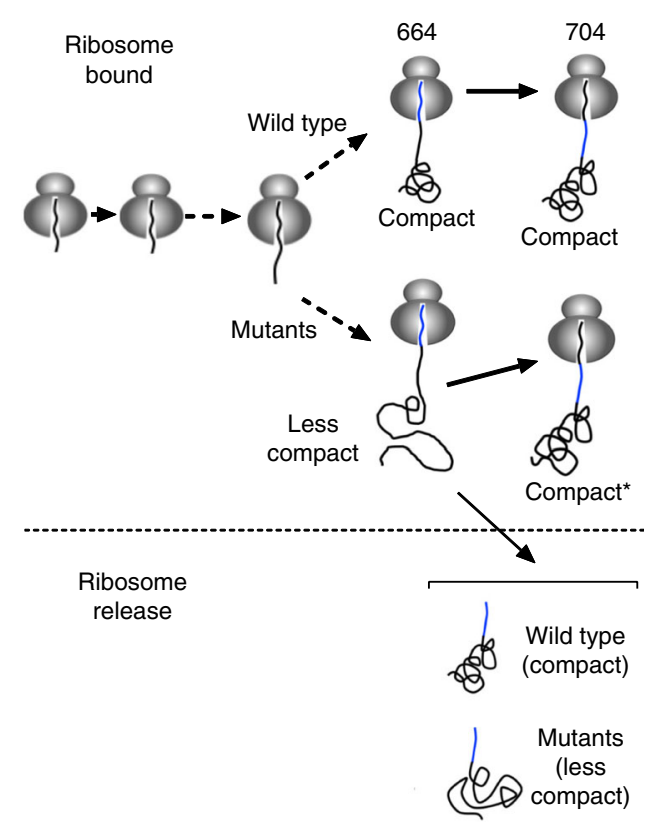

Fig. 3 Ribosome influences cotranslational folding defects. a, c FRET efficiencies of ribosome-bound and released CFP-NBD1 $\pm A 455 E$ or $\pm L 558 S$ polypeptides (acceptor dye at Arg487) truncated at residue 568 or $604 \mathbf{a}$, and 664 or $704 \mathbf{c}$. Each dot represents data from an independent experiment. Each bar shows mean \pm SEM,$n=3$ or 4 independent experiments. Two-tailed student's $t$ test, asterisk $p<0.05$, double asterisk $p<0.01$, triple asterisk $p<$ 0.001 , n.s. $>0.05$ otherwise indicated. Ribosome-released (RNase A) (truncation 568 or 664) and ribosome-bound (truncation 604 or 704 ) polypeptides contains equivalent cytosolically exposed residues, therefore differences in FRET are due to ribosome attachment. $\mathbf{b}$, $\mathbf{d}$ Illustration depicting results of $\mathbf{a}$, or c showing schematic of wild-type and mutant ribosome-bound and released polypeptides (acceptor dye at Arg487) truncated at indicated residue. b NBD1 polypeptides truncated at residue 568 remain unfolded on the ribosome and show an increase in FRET, consistent with $\alpha$-helical subdomain folding, following ribosome release. Wild-type ribosome-bound and released polypeptides are in the compact sate (high FRET) at truncation 604 , whereas mutant polypeptides remain less compact (low FRET) both before and after ribosome release. $\mathbf{d}$ Wild-type ribosome-bound and released polypeptides are shown in the compact sate at truncation 664, whereas mutant polypeptides remain less compact (low FRET) both before and after ribosome release. In contrast, both wild-type and mutant polypeptides truncated at residue 704 have achieved a compact state (high FRET). Source data of $\mathbf{a}$ and $\mathbf{b}$ are provided as a Source Data file.

the acceptor dye is attached to CFP by a short unstructured tether that is not dependent on NBD1 folding. In contrast, when the acceptor dye was inserted at Arg487 in NBD1 (truncation 654), an irreversible temperature-dependent decrease in FRET was observed (Supplementary Fig. 3d, e), consistent with previous studies showing that NBD1 does not spontaneously refold in vitro ${ }^{4,5}$. We then compared wild-type and mutant ribosomeattached polypeptides truncated at residue 654 and 744 . All constructs exhibited a decrease in FRET with increasing temperature, consistent with thermal denaturation from their respective folding states, and both mutants exhibited a lower FRET than wild-type at all temperatures tested $\left(4-50{ }^{\circ} \mathrm{C}\right.$, Fig. 4a-d). At the highest temperatures, differences between wildtype and mutants became less prominent. In addition, the NBD1folding intermediate (truncation 654), but not full-length NBD1, showed a slightly greater temperature dependence between wildtype and mutant polypeptides with a transition temperature above $35-40^{\circ} \mathrm{C}$ (Fig. 4e, f). Overall, temperature-dependent changes in FRET exhibited similar slopes and occurred over the same temperature range for wild-type and mutant polypeptides, 
a
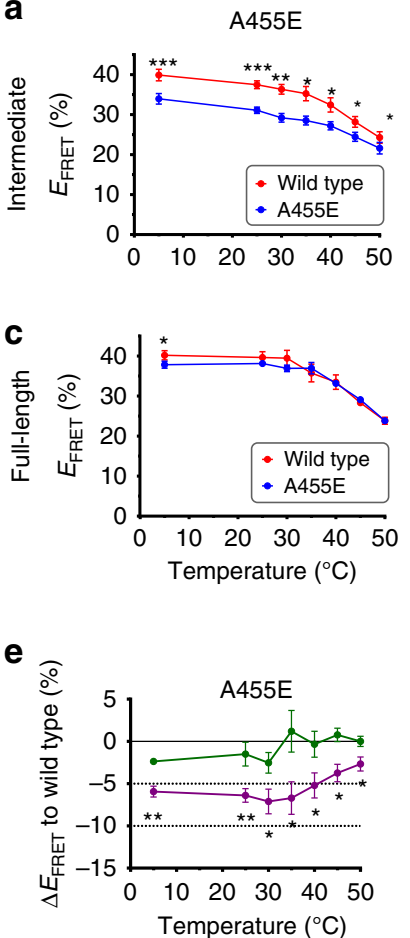

b

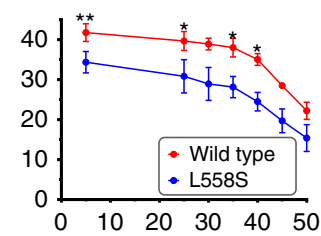

d

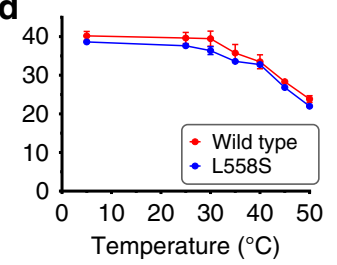

f

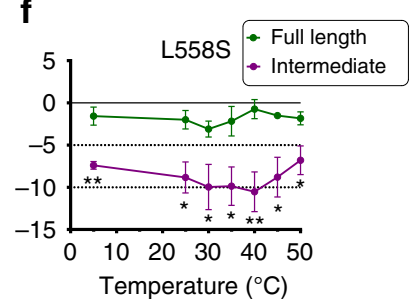

Fig. 4 Thermal stability of NBD1-folding intermediates. a-d Temperaturedependent FRET efficiencies of wild-type (red line) and A455E or L558S (blue lines) ribosome-bound CFP-NBD1 polypeptides. Acceptor dye was located at Arg487 and polypeptides were truncated at residue 654 (intermediate) $\mathbf{a}, \mathbf{b}$ or 744 (full-length) c, d. Data are mean \pm SEM, $n=3$ or 7 independent experiments. Two-tailed student's $t$ test, asterisk $p<0.05$, double asterisk $p<0.01$, triple asterisk $p<0.001$, n.s. $>0.05$ otherwise indicated. e, $\mathbf{f}$ Graphs show the difference between temperature-dependent FRET efficiencies for wild-type and mutants calculated from a-d. One-tailed student's $t$ test, ${ }^{\star} p<0.05,{ }^{\star \star} p<0.01$, n.s. $>0.05$ otherwise indicated. Note that wild-type data at $45^{\circ} \mathrm{C}$ in b-d, A455E data at $25,40,45$, and $50^{\circ} \mathrm{C}$ in c, and L558S data at $4,35,40,45$, and $50^{\circ} \mathrm{C}$ in $\mathbf{d}$ have error bars smaller than the dots. Source data of $\mathbf{a}-\mathbf{f}$ are provided as a Source Data file.

suggesting that the mutations change the conformational profile of the folding intermediate without a major change in thermal stability.

NBD1 transient folding defect is linked to CFTR trafficking. Finally, we applied a genetic approach to test whether restoring the cotranslational folding pathway was possible, and if so, whether it would correct the final folding outcome and restore CFTR trafficking. Here, we took advantage of the numerous suppressor mutations previously shown to improve F508del CFTR trafficking ${ }^{54,55}$. We specifically focused on a proline substitution at residue Ser492 (S492P), which is predicted to increase rigidity between the $\mathrm{N}$-terminal and the $\alpha$-helical subdomains ${ }^{56}$, and a well-known suppressor mutation I539T $^{57}$, which increases thermal stability of F508del NBD1.

As shown in Fig. 5a-c, introduction of S492P and I539T (PT) into NBD1 (truncation 654). completely restored FRET for A455E to the level observed for wild-type polypeptides but had no effect on the L558S mutant. In addition, combination of the PT suppressor with A455E restored thermal sensitivity of nascent polypeptides to a level that was indistinguishable from wild type (Fig. 5d, e). We then tested whether correction of the cotranslational folding effect would translate to the folding outcome of full-length CFTR. As shown in Fig. 5f, g,

a
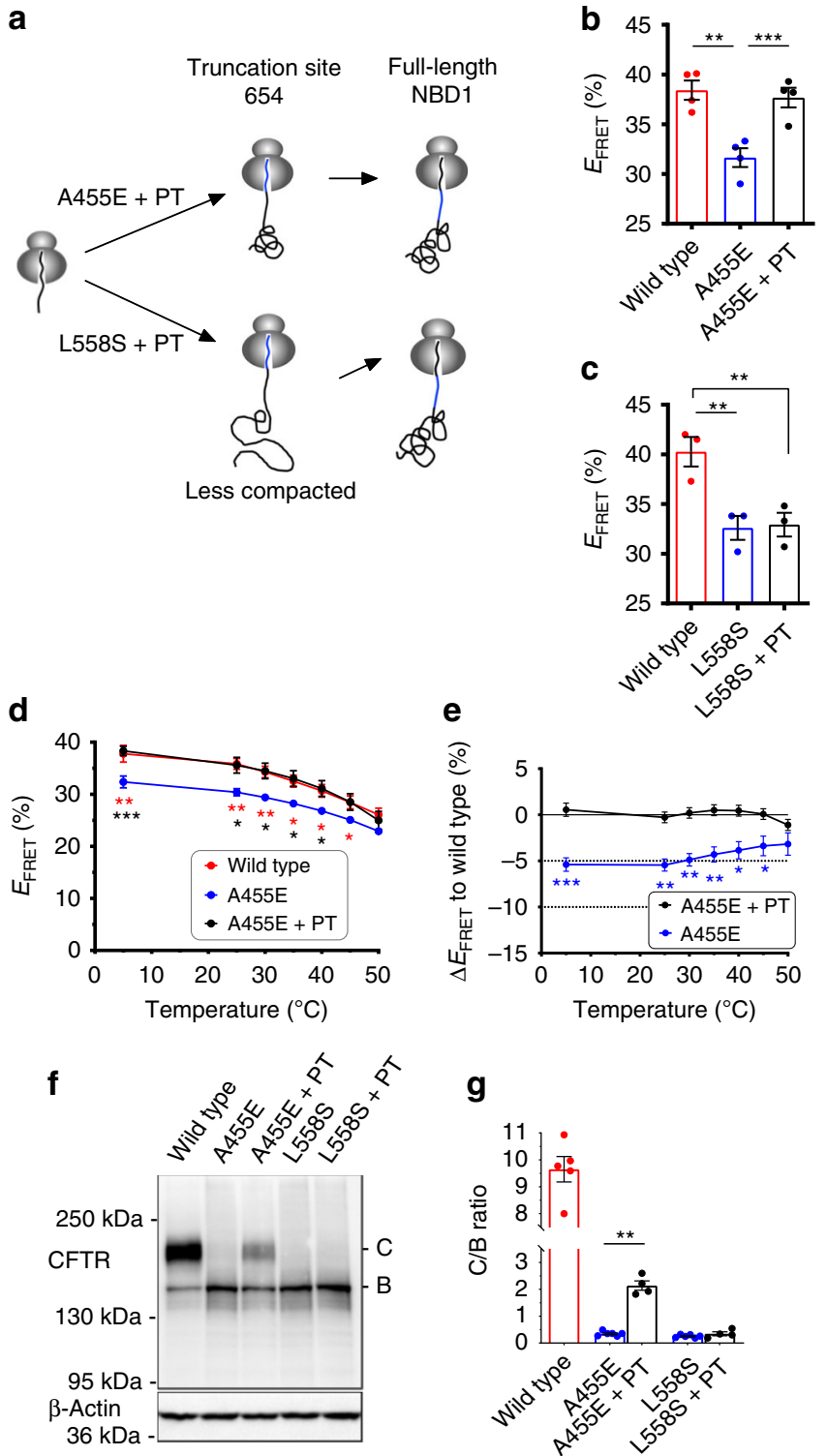

incorporation of the PT suppressor in full-length CFTR partially restored A455E CFTR trafficking in HEK293 cells as indicated by an increase in fully glycosylated band C protein to $20 \%$ of wildtype levels, whereas no improvement was observed in the processing of L558S CFTR. Partial restoration of A455E CFTR trafficking observed here is analogous to the effect of other suppressor mutations that effectively restore F508del NBD1 stability but have a relatively minor effect on CFTR trafficking $8,58,59$, and suggest that the cotranslational defect is not the only folding defect induced by the A455E mutation. Taken together, these results show that genetic complementation by suppressor mutations can overcome defects associated with transient folding intermediates and thereby partially restore the native folding landscape and final folding outcome.

\section{Discussion}

In this study, we show that disease-causing missense mutations in the CFTR chloride channel disrupt specific steps of the cotranslational folding pathway. Specifically, we demonstrate that (i) mutant NBD1-folding intermediates are less compact than their wild-type counterparts; (ii) two mutants, A455E and L558S, exert detectable effects during a brief window of synthesis that involves 
Fig. 5 Genetic correction of cotranslational folding also restores CFTR trafficking. a-c S492P \& 1539T (PT) suppressor mutations restore FRET efficiency of the folding intermediate (truncation at 654, acceptor dye at Arg487) for A455E b but not L558S c. Each dot represents data from an independent experiment. Each bar shows mean \pm SEM, $n=3$ or 4 independent experiments. Two-tailed student's $t$ test, asterisk $p<0.05$, double asterisk $p<0.01$, triple asterisk $p<0.001$, n.s. $>0.05$ otherwise indicated. d, e Temperature-dependent FRET efficiencies of wild-type (red line), A455E (blue line), and A455E + PT (black line). Ribosome-bound CFP-NBD1 polypeptides were truncated at residue 654 (acceptor dye at Arg487). The difference between wild-type and mutants in e were calculated from the data in $\mathbf{d}$. Data are mean $\pm \mathrm{SEM}, n=4$ independent experiments. Two-tailed student's $t$ test comparing wild-type and A455E (red asterisks), A455E and A455E + PT (black asterisks), or wild-type and $\mathrm{A} 455 \mathrm{E}+\mathrm{PT}$ (n.s.) in $\mathbf{d}$ and one-tailed student's $t$ test in $\mathbf{e}$, asterisk $p<0.05$, double asterisk $p<0.01$, n.s. $>0.05$ otherwise indicated. Note that some points have error bars smaller than the dot. $\mathbf{f}, \mathbf{g}$ Immunoblot of CFTR A455E or L558S and A455E or L558S plus PT mutations expressed in human embryonic kidney (HEK) 293 cells showing correction of folding defect for full-length CFTR A455E but not L558S. Uncropped blots in Source Data.

Each dot represents data from an independent experiment. Each bar shows mean \pm SEM, $n=4-6$ independent experiments. Two-tailed student's $t$ test, double asterisk $p<0.01$, n.s. $>0.05$ otherwise indicated. Source data of $\mathbf{b}-\mathbf{g}$ are provided as a Source Data file.

the coordinated folding of the $\alpha$-helical subdomain and the $\beta$ sheet core; (iii) the effect of ribosome attachment initially accentuates the folding defect by delaying $\alpha$-helical subdomain folding, and subsequently facilitates domain compaction as synthesis of NBD1 was completed; and (iv) genetic suppressor mutations that restore cotranslational folding also partially restore trafficking of full-length CFTR. These results indicate that disruption of protein folding by disease-causing mutations begins early during synthesis and suggest that transient folding intermediates provide potential targets for restoring folding in human disease.

The de novo protein folding landscape is characterized by folding intermediates, likely comprised of structural ensembles, that exist transiently and are sequentially remodeled as the nascent polypeptide progressively emerges from the ribosome exit tunnel ${ }^{17,18,24,47,60}$. With the caveat that some secondary structure (e.g., alpha-helices) may form within the ribosome exit tunnel $^{20,61,62}$, structure features of the nascent polypeptide are dictated primarily by the peptide region that becomes cytosolically exposed at any given length $17,18,60,63$. Consistent with this, the $\mathrm{N}$-terminal domain of HemK was shown to rapidly transition into a native-like structure after emerging from the ribosome ${ }^{27}$, consistent with results reported for $\beta$-barrel fluorescent proteins ${ }^{60}$. Molecular simulations of large single-domain proteins also predict that fast folding kinetics and/or transient translational pauses can improve cotranslational folding by avoiding kinetically stable non-native interactions with C-terminal residues ${ }^{64}$. Similarly, synonymous codon changes can enhance in vivo degradation without substantially changing activity/ function and thermal stability ${ }^{34}$. An extreme example reported by Sander et al. $^{32}$ used a tandem fluorescent protein approach wherein alternate stable structures were generated from the same polypeptide sequence based on the order in which cotranslational folding occurred. Therefore, the folding pathway is an important determinant of the final folded state. While it is well known that disease-causing mutations can impact the stability and/or structure of fully synthesized $\mathrm{CFTR}^{6,65}$, our results indicate that mutations can also exert their effect by disrupting intermediates along the folding pathway and thereby alter folding outcome.
The FRET-based approach used here provides a unique opportunity to evaluate transient folding intermediates, and several aspects of this system support our interpretation that changes in FRET reflect a conformational change in the structural ensemble of the nascent polypeptide ${ }^{48,49}$. First, all parameters of the experimental system were held constant with the exception of the mutant residue, and each experiment was carried out in parallel with the wild-type sequence to allow a direct comparison between wild-type and mutant nascent polypeptides. Second the absolute CFP fluorescence intensity (pps/nM) remained constant (as reported previously ${ }^{60}$ ) regardless of NBD1 chain length making it highly unlikely that the ribosome, the nascent NBD1 polypeptide, or other cellular factors affect FRET efficiency by altering CFP folding. Third, multiple sites for acceptor probe incorporation (described elsewhere) were evaluated and chosen so as not to grossly perturb NBD1 folding ${ }^{17,18}$. Overall, this approach enabled us to directly compare measurements made under identical experimental conditions where differences in FRET can be ascribed to the change of a single amino acid in the nascent polypeptide.

An important caveat of this system is that the readout for peptide compaction is limited to changes that can be detected by location of the donor and acceptor probes. The probe sites therefore restrict our ability to determine the precise nature of a structural defect and to identify changes that have minimal effect on the distance between these specific probes. Thus, the apparent resolution of the NBD1 folding defect observed at longer chain lengths as shown in Fig. 2 may be due to: (i) the inability of the probe sites to detect a residual folding abnormality ${ }^{17,18}$, (ii) a decrease in domain stability with minimal structural change, similar to that observed for crystal structures of F508del ${ }^{8,66}$ (albeit containing stabilizing site suppressor mutations), or (iii) subtle structural changes that may disrupt later folding events such as domain-domain assembly, which are known to be critical for CFTR trafficking ${ }^{2}$. It is also possible that in this system, the isolated wild-type NBD1 domain does not reach a completely folded native structure, thereby allowing FRET values to converge at longer chain lengths. Further studies using different approaches, for example NMR studies of nascent translation intermediates and/or high resolutions structures of A455E and L558S NBD1, will be needed to generate a more complete understanding of this process.

We do know, however, that NBD1 subdomains undergo a coordinated, stepwise folding process that is critical for NBD1 and full-length CFTR to achieve a mature conformation ${ }^{17,18}$. For example, the $\mathrm{N}$-terminal subdomain folds rapidly and independently, whereas folding of the $\alpha$-helical subdomain and $\beta$-sheet core is tightly coupled to translation and coordinated by biosynthetic machinery ${ }^{18}$. Previous studies using stalled ribosomeattached nascent chain complexes (RNCs) have shown that the ribosome can exert destabilizing effects on nascent polypeptide relative to the same peptide region free in aqueous solution ${ }^{22,28-30}$. For CFTR, the net result of this destabilizing effect is to delay compaction of the $\alpha$-helical subdomain until the $\beta$-sheet core is synthesized and thereby ensure that subdomains fold efficiently and in the proper temporal order to avoid offpathway intermediates ${ }^{18}$. These results are in good agreement with recent studies by Alexander et al. ${ }^{30}$, where ribosomemediated delay of calerythrin $\mathrm{N}$-terminal domain folding also facilitated overall folding efficiency.

Here, we show that the destabilizing effect of the ribosome exacerbates the disruptive effects of A455E and L558S mutations by further delaying $a$-helical subdomain folding (Fig. 2). Somewhat paradoxically, ribosome attachment also promoted and was required for domain compaction as NBD1 synthesis was completed (Fig. 3c, d). Thus the ribosome influences both normal and 
abnormal folding intermediates, and for A455E and L558S alters the normal progression (and timing) of NBD1 cotranslational folding events. It is important to consider that the kinetics of NBD1 de novo folding in the cell, relative to its native translation rate, could add an additional layer of complexity if the nascent folding intermediates are not in equilibrium during active peptide elongation ${ }^{30}$. In this context, the ribosome can cooperate with cellular chaperones to decelerate, accelerate and/or reverse domain folding to improve folding outcomes ${ }^{42}$. Reticulocyte lysate is an abundant source of HSP40, HSP70, HSP90, and other physiologic cellular chaperones that alter the rate of de novo folding and likely make an important contribution to our findings ${ }^{25,37-41,67,68}$. For technical reasons, we were not able to determine the effect of cellular chaperones on NBD1 folding in this study, and additional work is therefore needed to determine more precisely how these and other factors might influence the folding events studied here.

An unexpected finding was that NBD1 cotranslational folding defects were observed during a relatively brief window of synthesis, specifically as $\alpha$-helix $\mathrm{H} 5$ to $\beta$-strand $\mathrm{S} 7$ emerge from the ribosome, and were followed by domain compaction as the remaining NBD1 residues became available (Fig. 2). One possibility, given the very high contact order of $\mathrm{NBD} 1^{17,69}$, is that mutations of this nature exert their greatest disruptive effect when limited folding information (chain length) is available to stabilize nascent structure. The magnitude of the disruptive effect could therefore be decreased by stabilizing interactions that are provided by C-terminal residues during late stages of NBD1 synthesis $17,18,24,47,60$. Yet despite the apparent compaction of the domain as measured by FRET (Fig. 2c-f), some residual folding defect must still remain in the fulllength CFTR protein as demonstrated by its poor processing efficiency (Fig. 2a). Given the multidomain structure of CFTR, the precise nature of this folding defect is likely complex. PT suppressor mutations, which restore the in vitro cotranslational defect at short NBD1 chain lengths, only partially restore folding of the full-length protein in cells. Thus, in a manner analogous to F508del, cotranslational misfolding of A455E likely results in a local perturbation in NBD1 that is undetected by FRET, which impacts an additional subsequent alteration (e.g., domain-domain interactions) in the full-length protein.

CFTR processing is disrupted by multiple mutations that act via different mechanisms. For the most common variant, F508del, second site suppressor mutations have been identified that either stabilize NBD1 $1^{55,57,59}$ or correct defects in the NBD1-ICL4 interface. In each case, correcting a single defect only partially restores CFTR maturation in the cell ${ }^{6,8}$, and efficient maturation requires correcting both defects simultaneously ${ }^{7,8}$. This is also seen with pharmacological correction where the relatively weak effects of Lumacaftor and Tezacaftor, are greatly augmented by addition of a second corrector acting on a different folding defect. Consistent with these findings, PT suppressor mutations that correct the A455E NBD1 cotranslational folding defect only partially restore A455E CFTR trafficking in cells (Fig. 5), indicating that like F508del, A455E likely induces multiple folding defects. In addition, although A455E and L558S act at a similar stage of synthesis, PT mutations suppressed the cotranslational defect and restored CFTR trafficking only for A455E (Fig. 5), likely because introduction of a negatively charged residue into the NBD1 core (A455E) induces a different structural perturbation than a Leu-to-Ser substitution in the a-helical subdomain (L558S). The finding that both mutations act during the same window of synthesis, supports the previous hypothesis that folding of the $\alpha$-helical and $\beta$-sheet core is finely tuned and particularly sensitive to disruption ${ }^{18}$.

Taken together, our results indicate that cotranslational folding intermediates play an important role in the underlying mechanisms of de novo protein folding, and raise the possibility that they contribute to the molecular pathology of protein folding disorders. Although additional studies are needed to determine whether cotranslational folding intermediates might be druggable targets, genetic suppression of the cotranslational defect and the observed cotranslational effects of Lumacaftor on CFTR folding suggest that small molecules acting on such intermediates could potentially help restore CFTR processing. Thus, our findings may aid in the understanding and development of treatment approaches for small molecule correction in $\mathrm{CF}$ and other proteinfolding disorders.

\section{Methods}

Plasmids. pSP64-eCFP-NBD1 containing Amber stop codons (TAG) at CFTR residues Thr389, Arg487, and Asp567, pSP64-non-fluorescent CFP-NBD1 fusion constructs $^{17,18}$, and pcDNA3-CFTR ${ }^{52}$ were used in this study. For cloning, CFcausing mutations (L441P, A455E, S492F, I507del, F508del, V520F, G551D, L558S A559T, R560K, and R560T), a non-disease-causing mutation M470V, and suppressor mutations (S492P and I539T) were individually introduced into pSP64eCFP-NBD1-R487TAG ${ }^{17,18}$, pSP64-eCFP-NBD1-D567TAG ${ }^{17,18}$, or pcDNA3$\mathrm{CFTR}^{52}$ by PCR overlap extension using complementary sense and antisense oligonucleotides (primers\# 1-28) listed in Supplementary Table 1. The CFTR2 database (https://www.cftr2.org) was referred to design the oligonucleotides. PCR fragment containing the mutation L441P, A455E, M470V, S492F, I507del, F508del, or V520F was subcloned into PstI and SphI sites of pSP64-eCFP-NBD1-R487TAG/ D567TAG ${ }^{17,18}$. PCR fragment containing the mutation L558S, A559T, R560K, or R560T was subcloned into SphI and SpeI sites of pSP64-eCFP-NBD1-R487TAG/ D567TAG ${ }^{17,18}$. PCR fragment containing the mutations S492P and I539T (PT) was subcloned into PstI and SpeI sites of pSP64-eCFP-NBD1-R487TAG ${ }^{17,18}$. PCR fragment containing the mutations A455E, L558S, A455E + PT, or L558S + PT was subcloned into BsrGI and HpaI sites of pcDNA3-CFTR ${ }^{52}$. All cloned PCR fragments were verified by DNA sequencing. Truncated cDNA for in vitro transcription was generated by PCR amplification using appropriate oligonucleotides (primers\# 29-41) listed in Supplementary Table 2.

In vitro transcription and translation. Noncapped RNA transcripts were synthesized from PCR-amplified DNA templates $(15 \mathrm{ng} / \mu \mathrm{l})$ in a solution containing $80 \mathrm{mM}$ HEPES-NaOH (pH 7.5); $16 \mathrm{mM} \mathrm{MgCl}_{2} ; 2 \mathrm{mM}$ spermidine; $3 \mathrm{mM}$ each ATP, CTP, UTP, and GTP; $10 \mathrm{mM}$ DTT; $0.2 \mathrm{U} / \mu \mathrm{l}$ RNase inhibitor; and $5 \mu \mathrm{g} / \mathrm{ml}$ SP6 RNA polymerase at $40^{\circ} \mathrm{C}$ for $2 \mathrm{~h}$. RNA was precipitated with $3 \mathrm{M} \mathrm{LiCl}$ and $20 \mathrm{mM}$ ethylenediaminetetraacetic acid (EDTA) at $-20^{\circ} \mathrm{C}$ for $1 \mathrm{~h}$ and washed three times with $70 \%(\mathrm{v} / \mathrm{v})$ ethanol, followed by centrifugation at $16,000 \times g$ at $4{ }^{\circ} \mathrm{C}$ The RNA pellet was resuspended in RNase free $\mathrm{H}_{2} \mathrm{O}$ and stored at $-80{ }^{\circ} \mathrm{C}$.

Four $250 \mu \mathrm{l}$ in vitro translation reactions were prepared in parallel in reactions containing $60 \mathrm{ng} / \mu \mathrm{l}$ purified RNA, 40\% rabbit reticulocyte lysate (RRL), $20 \mathrm{mM}$ HEPES-KOH (pH 7.6), $100 \mathrm{mM} \mathrm{KOAc,} \mathrm{1.6-2.0} \mathrm{mM} \mathrm{Mg(OAc)} 2,50 \mu \mathrm{M}$ each of 20 amino acids, $1 \mathrm{mM}$ ATP, $1 \mathrm{mM}$ GTP, $15 \mathrm{mM}$ creatine phosphate, $2 \mathrm{mM}$ DTT, $0.15 \mathrm{mM}$ spermidine, $20 \mathrm{ng} / \mu \mathrm{l}$ bovine tRNA, $40 \mathrm{ng} / \mu \mathrm{l}$ creatine kinase, $0.12 \mathrm{U} / \mu \mathrm{l}$ RNase inhibitor, $0.2-2 \mu \mathrm{M}$ RNA aptamer ${ }^{70}$, and $1 \mu \mathrm{M}$ synthetic amber suppressor tRNA (either $\left[{ }^{14} \mathrm{C}\right]$ Lys-tRNA ${ }^{\mathrm{amb}}$ or $\varepsilon N-7$-nitrobenz-2-oxa-1,3-diazol ( $\varepsilon N B D$ )$\left[{ }^{14} \mathrm{C}\right]$ Lys-tRNA $\left.{ }^{\mathrm{amb}}\right)^{17}$. To generate matched samples for fluorescence spectroscopy, CFP donor only (D)-translated in the presence of $\left[{ }^{14} \mathrm{C}\right]$ Lys-tRNA ${ }^{\mathrm{amb}}$, Donor + Acceptor $(\mathrm{D}+\mathrm{A})$-translated in the presence of $\varepsilon N B D-\left[{ }^{14} \mathrm{C}\right]$ Lys-tRNA ${ }^{\mathrm{amb}}$, and two "blank" (control) reactions using non-fluorescence eCFP lacking a UAG codon translated in the presence of either $\left[{ }^{14} \mathrm{C}\right]$ Lys-tRNA ${ }^{a m b}$ or $\varepsilon N B D-\left[{ }^{14} \mathrm{C}\right] \mathrm{Lys}-$

$\mathrm{tRNA}^{\mathrm{amb}}$ (BD and BDA) were prepared as described in Supplementary Figure $1 \mathrm{~b}$. Blank samples were used to quantify the concentration of RNCs and obtain ${ }^{14} \mathrm{C}$ corrected fluorescence intensity in solution-based FRET measurement. An RNA aptamer that inhibits translation termination factors (eRF1/eRF3) was added $(0.2-2 \mu \mathrm{M})$ to achieve similar read-through efficiencies of the UAG codon for D and $\mathrm{D}+\mathrm{A}$ translation reactions ${ }^{18,70}$. Translation reactions were incubated at $24^{\circ} \mathrm{C}$ for $8 \mathrm{~min}$ prior to addition mRNA, suppressor tRNA, and RNA aptamer, and then at $24^{\circ} \mathrm{C}$ for $72 \mathrm{~min}$ (to enable CFP folding and maturation ${ }^{60}$ ).

Compensation for effects of in vitro translational stalls. If read-through efficiency at the UAG codon differs for $\mathrm{D}$ and $\mathrm{D}+\mathrm{A}$ samples in single experiments, then stalled RNCs in D and D + A samples can introduce affect in FRET. This is because the relative fraction of stalled polypeptides (with zero FRET) in the sample, is dependent on both the number of stalls and the number of polypeptides that actually read-through the stop codon ${ }^{18}$. To eliminate this potential artifact, only samples with D/DA ratio between 0.80 and 1.20 , or 0.85 and 1.15 were used for R487TAG or D567TAG constructs, respectively.

Fluorescence measurements. Intact RNCs were isolated from translation reactions at $4{ }^{\circ} \mathrm{C}$ by size exclusion column chromatography (Sepharose CL-6B) equilibrated in buffer containing $40 \mathrm{mM}$ HEPES-KOH (pH 7.6), $100 \mathrm{mM} \mathrm{KOAc}$, and 
$10 \mathrm{mM} \mathrm{MgCl}_{2}$, and the ribosome concentration $\left(\mathrm{A}_{260}\right)$ in each sample was normalized by addition of buffer. CFP fluorescence emission spectra $(\lambda e x=430 \mathrm{~nm}$, $\lambda \mathrm{em}=450-600 \mathrm{~nm}, 1 \mathrm{~nm}$ intervals) obtained from purified RNCs were measured using a Fluorolog 3-22 fluorometer (HORIBA Jobin Yvon, Edison, NJ) at $25^{\circ} \mathrm{C}$ unless otherwise specified. The five highest peak emission intensities $(\sim 475 \mathrm{~nm})$ were averaged and used for subsequent calculation. Ribosome release was performed by adding $200 \mu \mathrm{g} / \mathrm{ml}$ RNase A in column buffer plus $3 \mathrm{mM} \mathrm{ATP}$, which effectively releases all nascent chains from the ribosome with $2 \mathrm{~min}^{17}$. At the end of measurement, the concentration of polypeptide in $\mathrm{D}$ and $\mathrm{D}+\mathrm{A}$ samples was determined by ${ }^{14} \mathrm{C}$ scintillation counting using the following equation:

$$
[\mathrm{RNC}]=\left(\mathrm{cpm}_{\mathrm{S}}-\mathrm{cpm}_{\mathrm{B}}\right) /(\mathrm{CE} x \mathrm{SA} x \mathrm{vol})
$$

where [RNC] is the concentration of RNCs (in $\mathrm{nM}$ ), $\mathrm{cpm}_{\mathrm{S}}$ is ${ }^{14} \mathrm{C}$ counts/min of D or $\mathrm{D}+\mathrm{A}$ sample, and $\mathrm{cpm}_{\mathrm{B}}$ is ${ }^{14} \mathrm{C}$ counts/min of blank (BD or $\mathrm{BDA}$ ), respectively. CE is counting efficiency (estimated at $95 \%$ for ${ }^{14} \mathrm{C}$ ), SA is the specific activity of $\left[{ }^{14} \mathrm{C}\right] \mathrm{Lys}$ in $\mathrm{dpm} / \mathrm{pmol}$, and vol is the volume of sample in $\mathrm{ml}$.

FRET efficiency was then calculated by the acceptor-dependent decrease in CFP fluorescence intensity, from the following equation:

$$
\mathrm{E}_{\mathrm{FRET}}=1-\mathrm{F}_{\mathrm{DA}} / \mathrm{F}_{\mathrm{D}}
$$

where $\mathrm{F}_{\mathrm{DA}}$ and $\mathrm{F}_{\mathrm{D}}$ are the net ${ }^{14} \mathrm{C}$-corrected fluorescence intensity per nM nascent chain polypeptide in $\mathrm{D}+\mathrm{A}$ and $\mathrm{D}$ samples, respectively. Note that fluorescence intensity of the acceptor is negligible in our sample (under these conditions) ${ }^{17,18}$.

Each experiment was performed with a matched pair of wild-type and mutant constructs, therefore, controlling for day-to-day variation in FRET efficiency, and allowing a direct comparison of differences in FRET efficiency between wild-type and mutant constructs to be evaluated directly at specific stage of synthesis.

Immunoblotting. Human embryonic kidney (HEK) 293 cells (ATCC, catalog\# CRL-1573) were grown at $37^{\circ} \mathrm{C}$ under $5 \% \mathrm{CO}_{2}$ in Dulbecco's modified Eagle's medium (Invitrogen) supplemented with $10 \%$ fetal calf serum (Thermo Scientific) and penicillin/streptomycin (Invitrogen). In total, $5 \times 10^{5}$ cells were seeded in sixwell plates and transfected 1 day later with equal amounts $(2.5 \mu \mathrm{g})$ of pcDNA3CFTR vector. Cells were harvested $48 \mathrm{~h}$ after transfection by lysis for $20 \mathrm{~min}$ in $600 \mu \mathrm{l}$ of ice-cold RIPA buffer (20 mM HEPES-NaOH/pH 7.5, $150 \mathrm{mM} \mathrm{NaCl}$, $1 \mathrm{mM}$ EDTA, $1 \%$ Triton X-100, $0.1 \%$ SDS, $0.5 \%$ sodium deoxycholate) containing complete protease inhibitor mixture (Roche Applied Science). Cell lysate was separated by sodium dodecyl sulfate polyacrylamide gel electrophoresis, transferred to polyvinylidene difluoride membrane (Millipore), and immunoblotted using the following primary antibodies: (1) mouse anti-CFTR M3A7 (Millipore, Cat\# 05-593, Lot\# 2652963, 1:2000 dilution), or (2) rabbit anti- $\beta$-actin C4 (Santa Cruz Biotech., Cat\# sc-47778, Lot\# B1914, 1:2000 dilution) and secondary antibodies: (3) goat anti-mouse IgG (H + L)-HRP conjugate (Bio-Rad, Cat\# 1706516, 1:5000 dilution), or (4) goat anti-rabbit IgG-HRP (Santa Cruz Biotech., Cat\# sc-2030, 1:5000 dilution). Blots were imaged using the ChemiDoc XRS + System (Bio-Rad) and analyzed using accompanying image analysis software. Uncropped blots are available in the Source Data file.

Reporting summary. Further information on research design is available in the Nature Research Reporting Summary linked to this article.

\section{Data availability}

Data supporting the findings of this manuscript are available from the corresponding author upon reasonable request. A reporting summary for this Article is available as a Supplementary Information file. Source data are provided with this paper.

Received: 15 August 2018; Accepted: 4 August 2020;

Published online: 26 August 2020

\section{References}

1. Cutting, G. R. Cystic fibrosis genetics: from molecular understanding to clinical application. Nat. Rev. Genet. 16, 45-56 (2015).

2. Sosnay, P. R. et al. Defining the disease liability of variants in the cystic fibrosis transmembrane conductance regulator gene. Nat. Genet. 45, 1160-1167 (2013).

3. Lukacs, G. L. \& Verkman, A. S. CFTR: folding, misfolding and correcting the DeltaF508 conformational defect. Trends Mol. Med. 18, 81-91 (2012).

4. Protasevich, I. et al. Thermal unfolding studies show the disease causing F508del mutation in CFTR thermodynamically destabilizes nucleotidebinding domain 1. Protein Sci. 19, 1917-1931 (2010).

5. Wang, C. et al. Integrated biophysical studies implicate partial unfolding of NBD1 of CFTR in the molecular pathogenesis of F508del cystic fibrosis. Protein Sci. 19, 1932-1947 (2010).
6. Thibodeau, P. H. et al. The cystic fibrosis-causing mutation deltaF508 affects multiple steps in cystic fibrosis transmembrane conductance regulator biogenesis. J. Biol. Chem. 285, 35825-35835 (2010).

7. Rabeh, W. M. et al. Correction of both NBD1 energetics and domain interface is required to restore DeltaF508 CFTR folding and function. Cell 148, 150-163 (2012).

8. Mendoza, J. L. et al. Requirements for efficient correction of DeltaF508 CFTR revealed by analyses of evolved sequences. Cell 148, 164-174 (2012).

9. Mijnders, M., Kleizen, B. \& Braakman, I. Correcting CFTR folding defects by small-molecule correctors to cure cystic fibrosis. Curr. Opin. Pharm. 34, 83-90 (2017).

10. Taylor-Cousar, J. L. et al. Tezacaftor-ivacaftor in patients with cystic fibrosis homozygous for Phe508del. N. Engl. J. Med. 377, 2013-2023 (2017).

11. Boyle, M. P. et al. A CFTR corrector (lumacaftor) and a CFTR potentiator (ivacaftor) for treatment of patients with cystic fibrosis who have a phe508del CFTR mutation: a phase 2 randomised controlled trial. Lancet Respir. Med 2, 527-538 (2014)

12. Middleton, P. G. et al. Elexacaftor-tezacaftor-ivacaftor for cystic fibrosis with a single Phe508del allele. N. Engl. J. Med 381, 1809-1819 (2019).

13. Heijerman, H. G. M. et al. Efficacy and safety of the elexacaftor plus tezacaftor plus ivacaftor combination regimen in people with cystic fibrosis homozygous for the F508del mutation: a double-blind, randomised, phase 3 trial. Lancet 394, 1940-1948 (2019).

14. Mitraki, A., Fane, B., Haase-Pettingell, C., Sturtevant, J. \& King, J. Global suppression of protein folding defects and inclusion body formation. Science 253, 54-58 (1991)

15. Okiyoneda, T. et al. Mechanism-based corrector combination restores DeltaF508-CFTR folding and function. Nat. Chem. Biol. 9, 444-454 (2013).

16. Clark, P. L. Protein folding in the cell: reshaping the folding funnel. Trends Biochem Sci. 29, 527-534 (2004).

17. Khushoo, A., Yang, Z., Johnson, A. E. \& Skach, W. R. Ligand-driven vectorial folding of ribosome-bound human CFTR NBD1. Mol. Cell 41, 682-692 (2011).

18. Kim, S. J. et al. Protein folding. Translational tuning optimizes nascent protein folding in cells. Science 348, 444-448 (2015).

19. Nissley, D. A. et al. Accurate prediction of cellular co-translational folding indicates proteins can switch from post- to co-translational folding. Nat. Commun. 7, 10341 (2016).

20. Nilsson, O. B. et al. Cotranslational folding of spectrin domains via partially structured states. Nat. Struct. Mol. Biol. 24, 221-225 (2017).

21. Wruck, F., Katranidis, A., Nierhaus, K. H., Buldt, G. \& Hegner, M. Translation and folding of single proteins in real time. Proc. Natl Acad. Sci. USA 114, E4399-E4407 (2017).

22. Samelson, A. J., Jensen, M. K., Soto, R. A., Cate, J. H. \& Marqusee, S. Quantitative determination of ribosome nascent chain stability. Proc. Natl Acad. Sci. USA 113, 13402-13407 (2016).

23. Samelson, A. J. et al. Kinetic and structural comparison of a protein's cotranslational folding and refolding pathways. Sci. Adv. 4, eaas9098 (2018).

24. Thommen, M., Holtkamp, W. \& Rodnina, M. V. Co-translational protein folding: progress and methods. Curr. Opin. Struct. Biol. 42, 83-89 (2017).

25. Waudby, C. A., Dobson, C. M. \& Christodoulou, J. Nature and regulation of protein folding on the ribosome. Trends Biochem Sci. 44, 914-926 (2019).

26. Kramer, G., Shiber, A. \& Bukau, B. Mechanisms of cotranslational maturation of newly synthesized proteins. Annu Rev. Biochem 88, 337-364 (2019).

27. Holtkamp, W. et al. Cotranslational protein folding on the ribosome monitored in real time. Science 350, 1104-1107 (2015).

28. Kaiser, C. M., Goldman, D. H., Chodera, J. D., Tinoco, I. Jr. \& Bustamante, C. The ribosome modulates nascent protein folding. Science 334, 1723-1727 (2011).

29. Cabrita, L. D. et al. A structural ensemble of a ribosome-nascent chain complex during cotranslational protein folding. Nat. Struct. Mol. Biol. 23, 278-285 (2016).

30. Alexander, L. M., Goldman, D. H., Wee, L. M. \& Bustamante, C. Nonequilibrium dynamics of a nascent polypeptide during translation suppress its misfolding. Nat. Commun. 10, 2709 (2019).

31. Buhr, F. et al. Synonymous codons direct cotranslational folding toward different protein conformations. Mol. Cell 61, 341-351 (2016).

32. Sander, I. M., Chaney, J. L. \& Clark, P. L. Expanding Anfinsen's principle: contributions of synonymous codon selection to rational protein design. J. Am. Chem. Soc. 136, 858-861 (2014).

33. Stein, K. C. \& Frydman, J. The stop-and-go traffic regulating protein biogenesis: How translation kinetics controls proteostasis. J. Biol. Chem. 294, 2076-2084 (2019)

34. Walsh, I. M., Bowman, M. A., Soto Santarriaga, I. F., Rodriguez, A. \& Clark, P L. Synonymous codon substitutions perturb cotranslational protein folding in vivo and impair cell fitness. Proc. Natl Acad. Sci. USA 117, 3528-3534 (2020).

35. McGuffee, S. R. \& Elcock, A. H. Diffusion, crowding \& protein stability in a dynamic molecular model of the bacterial cytoplasm. PLoS Comput Biol. 6, e1000694 (2010). 
36. Elcock, A. H. Models of macromolecular crowding effects and the need for quantitative comparisons with experiment. Curr. Opin. Struct. Biol. 20, 196-206 (2010).

37. Willmund, F. et al. The cotranslational function of ribosome-associated Hsp70 in eukaryotic protein homeostasis. Cell 152, 196-209 (2013).

38. Wruck, F. et al. Protein folding mediated by trigger factor and Hsp70: new insights from single-molecule approaches. J. Mol. Biol. 430, 438-449 (2017).

39. Gumiero, A. et al. Interaction of the cotranslational Hsp70 Ssb with ribosomal proteins and rRNA depends on its lid domain. Nat. Commun. 7, 13563 (2016).

40. Doring, K. et al. Profiling Ssb-nascent chain interactions reveals principles of Hsp70-assisted folding. Cell 170, 298-311 e20 (2017).

41. Stein, K. C., Kriel, A. \& Frydman, J. Nascent polypeptide domain topology and elongation rate direct the cotranslational hierarchy of Hsp70 and TRiC/CCT. Mol. Cell 75, 1117-1130 e5 (2019).

42. Liu, K., Maciuba, K. \& Kaiser, C. M. The ribosome cooperates with a chaperone to guide multi-domain protein folding. Mol. Cell 74, 310-319 e7 (2019).

43. Kim, S. J. \& Skach, W. R. Mechanisms of CFTR folding at the endoplasmic reticulum. Front Pharm. 3, 201 (2012)

44. Mendoza, J. L. \& Thomas, P. J. Building an understanding of cystic fibrosis on the foundation of $\mathrm{ABC}$ transporter structures. J. Bioenerg. Biomembr. 39, 499-505 (2007).

45. Gee, H. Y. et al. The L441P mutation of cystic fibrosis transmembrane conductance regulator and its molecular pathogenic mechanisms in a Korean patient with cystic fibrosis. J. Korean Med. Sci. 25, 166-171 (2010).

46. Hou, B., Lin, P. J. \& Johnson, A. E. Membrane protein TM segments are retained at the translocon during integration until the nascent chain cues FRET-detected release into bulk lipid. Mol. Cell 48, 398-408 (2012).

47. Devaraneni, P. K. et al. Stepwise insertion and inversion of a type II signal anchor sequence in the ribosome-Sec61 translocon complex. Cell 146, 134-147 (2011).

48. Woolhead, C. A., McCormick, P. J. \& Johnson, A. E. Nascent membrane and secretory proteins differ in FRET-detected folding far inside the ribosome and in their exposure to ribosomal proteins. Cell 116, 725-736 (2004).

49. Nissley, D. A. \& O'Brien, E. P. Structural origins of FRET-observed nascent chain compaction on the ribosome. J. Phys. Chem. B 122, 9927-9937 (2018).

50. Eichmann, C., Preissler, S., Riek, R. \& Deuerling, E. Cotranslational structure acquisition of nascent polypeptides monitored by NMR spectroscopy. Proc. Natl Acad. Sci. USA 107, 9111-9116 (2010).

51. Cabrita, L. D., Hsu, S. T., Launay, H., Dobson, C. M. \& Christodoulou, J. Probing ribosome-nascent chain complexes produced in vivo by NMR spectroscopy. Proc. Natl Acad. Sci. USA 106, 22239-22244 (2009).

52. Matsumura, Y., David, L. L. \& Skach, W. R. Role of Hsc70 binding cycle in CFTR folding and endoplasmic reticulum-associated degradation. Mol. Biol. Cell 22, 2797-2809 (2011)

53. Carlson, E., Bays, N., David, L. \& Skach, W. R. Reticulocyte lysate as a model system to study endoplasmic reticulum membrane protein degradation. Methods Mol. Biol. 301, 185-205 (2005).

54. Aleksandrov, L. A. et al. Thermal stability of purified and reconstituted CFTR in a locked open channel conformation. Protein Expr. Purif. 116, 159-166 (2015).

55. Aleksandrov, A. A. et al. Allosteric modulation balances thermodynamic stability and restores function of DeltaF508 CFTR. J. Mol. Biol. 419, 41-60 (2012).

56. Estacio, S. G., Martiniano, H. F. \& Faisca, P. F. Thermal unfolding simulations of NBD1 domain variants reveal structural motifs associated with the impaired folding of F508del-CFTR. Mol. Biosyst. 12, 2834-2848 (2016).

57. Hoelen, H. et al. The primary folding defect and rescue of DeltaF508 CFTR emerge during translation of the mutant domain. PLoS ONE 5, e15458 (2010).

58. He, L. et al. Restoration of domain folding and interdomain assembly by second-site suppressors of the DeltaF508 mutation in CFTR. FASEB J. 24, 3103-3112 (2010).

59. DeCarvalho, A. C., Gansheroff, L. J. \& Teem, J. L. Mutations in the nucleotide binding domain 1 signature motif region rescue processing and functional defects of cystic fibrosis transmembrane conductance regulator delta f508. J. Biol. Chem. 277, 35896-35905 (2002).

60. Kelkar, D. A., Khushoo, A., Yang, Z. \& Skach, W. R. Kinetic analysis of ribosome-bound fluorescent proteins reveals an early, stable, cotranslational folding intermediate. J. Biol. Chem. 287, 2568-2578 (2012).

61. Daniel, C. J., Conti, B., Johnson, A. E. \& Skach, W. R. Control of translocation through the Sec61 translocon by nascent polypeptide structure within the ribosome. J. Biol. Chem. 283, 20864-20873 (2008).

62. Kosolapov, A. \& Deutsch, C. Tertiary interactions within the ribosomal exit tunnel. Nat. Struct. Mol. Biol. 16, 405-411 (2009).

63. Notari, L., Martinez-Carranza, M., Farias-Rico, J. A., Stenmark, P. \& von Heijne, G. Cotranslational folding of a pentarepeat betahelix protein. J. Mol. Biol. 430, 5196-5206 (2018).
64. Bitran, A., Jacobs, W. M., Zhai, X. \& Shakhnovich, E. Cotranslational folding allows misfolding-prone proteins to circumvent deep kinetic traps. Proc. Natl Acad. Sci. USA 117, 1485-1495 (2020).

65. Du, K., Sharma, M. \& Lukacs, G. L. The DeltaF508 cystic fibrosis mutation impairs domain-domain interactions and arrests post-translational folding of CFTR. Nat. Struct. Mol. Biol. 12, 17-25 (2005).

66. Lewis, H. A. et al. Impact of the deltaF508 mutation in first nucleotide-binding domain of human cystic fibrosis transmembrane conductance regulator on domain folding and structure. J. Biol. Chem. 280, 1346-1353 (2005).

67. Coppinger, J. A. et al. A chaperone trap contributes to the onset of cystic fibrosis. PLoS ONE 7, e37682 (2012).

68. Bagdany, M. et al. Chaperones rescue the energetic landscape of mutant CFTR at single molecule and in cell. Nat. Commun. 8, 398 (2017).

69. Plaxco, K. W., Simons, K. T. \& Baker, D. Contact order, transition state placement and the refolding rates of single domain proteins. J. Mol. Biol. 277, 985-994 (1998).

70. Gubbens, J., Kim, S. J., Yang, Z., Johnson, A. E. \& Skach, W. R. In vitro incorporation of nonnatural amino acids into protein using tRNA(Cys) derived opal, ochre, and amber suppressor tRNAs. RNA 16, 1660-1672 (2010).

\section{Acknowledgements}

We thank members of the CFFT Lab for helpful discussions, and Nikolay V. Dokholyan, David D. Mowrey, Venkat R. Chirasani, Konstantin I. Popov, and Jian Wang for sharing results of molecular dynamic simulations. This research was funded by the Cystic Fibrosis Foundation, NIH grants R01GM53457 and R01DK51818 (to WRS), Cystic Fibrosis Foundation Therapeutics Inc. grant SKACH05X0 (to WRS), and American Lung Association grant RT-269559-N (to HS).

\section{Author contributions}

H.S. designed and executed the series of experiments, analyzed results and wrote the manuscript. J.S.Y. designed and performed immunoblotting experiments and assisted in writing the manuscript. Z.Y. designed and carried out molecular biology experiments. W.R.S. conceived the project, designed experiments, and assisted in writing the manuscript.

\section{Competing interests}

H.S., J.Y.S., and W.R.S. are employees of the Cystic Fibrosis Foundation, which is involved in and funding independent programs for CF drug development including CFTR modulators that improve CFTR folding. The authors declare no personal competing financial and non-financial interests with Vertex Pharmaceuticals, but acknowledge that as a result of financial support, CFF may be entitled to receive royalty payments from Vertex for marketed products.

\section{Additional information}

Supplementary information is available for this paper at https://doi.org/10.1038/s41467020-18101-8.

Correspondence and requests for materials should be addressed to W.R.S

Peer review information Nature Communications Patricia Faisca and other, anonymous, reviewers for their contributions to the peer review of this work. Peer review reports are available.

Reprints and permission information is available at http://www.nature.com/reprints

Publisher's note Springer Nature remains neutral with regard to jurisdictional claims in published maps and institutional affiliations.

Open Access This article is licensed under a Creative Commons Attribution 4.0 International License, which permits use, sharing, adaptation, distribution and reproduction in any medium or format, as long as you give appropriate credit to the original author(s) and the source, provide a link to the Creative Commons license, and indicate if changes were made. The images or other third party material in this article are included in the article's Creative Commons license, unless indicated otherwise in a credit line to the material. If material is not included in the article's Creative Commons license and your intended use is not permitted by statutory regulation or exceeds the permitted use, you will need to obtain permission directly from the copyright holder. To view a copy of this license, visit http://creativecommons.org/ licenses/by/4.0\%

(C) The Author(s) 2020 